\title{
Reduced Dengue Incidence During COVID-19 Restrictions in Sri Lanka From March 2020 to April 2021
}

Sinnathamby Noble Surendran ( $\nabla$ noble@univ.jfn.ac.lk)

University of Jaffna

Ratnarajah Nagulan

University of Jaffna

Kokila Sivabalakrishnan

University of Jaffna

\section{Sivasingham Arthiyan}

University of Jaffna

Annathurai Tharshan

University of Jaffna

Thirunavukkarasu Jeyadas

University of Jaffna

Selvarajah Raveendran

University of Jaffna

\section{T Kumanan}

University of Jaffna

\section{Ranjan Ramasamy}

University of Jaffna

\section{Research Article}

Keywords: Aedes vectors, COVID-19 containment measures, dengue incidence, Jaffna district, Sri Lanka, vector control

Posted Date: September 24th, 2021

DOI: https://doi.org/10.21203/rs.3.rs-609645/v1

License: (c) (i) This work is licensed under a Creative Commons Attribution 4.0 International License. Read Full License 


\section{Abstract}

\section{Background}

Dengue is a major public health concern in Sri Lanka. COVID-19 in Sri Lanka was first detected in January 2020, and has continued to be prevalent in the country since that time. The impact of public health measures imposed to restrict COVID-19 transmission on the incidence of dengue throughout the island and particularly its northern Jaffna district in the period March 2020 to April 2021 was determined.

\section{Methods}

The incidence of dengue and COVID-19, rainfall and the public health measures implemented to contain COVID-19 transmission for each district in Sri Lanka were obtained from Government sources. The Seasonal Autoregressive Integrated Moving Average (SARIMA) model was used to predict the dengue incidence expected in March 2020 to April 2021 , based on pre-pandemic data and this was compared with the actual reported incidence of dengue during the period of COVID-19 restrictions. Ovitrap collections of Aedes larvae were also carried out in the Gurunagar ward of Jaffna city in the Jaffna district during the 2020 and 2021 lockdown and the findings compared with data from 2019.

\section{Results}

The reported number of dengue cases for the whole country from March 2020 to April 2021 was significantly lower than the numbers of dengue cases predicted from the five years immediately preceding the COVID-19 pandemic (2015-2019). Decreased numbers of dengue cases were reported compared to predicted numbers of cases in all 25 administrative districts in the country including the Jaffna district. Aedes larval numbers collected from ovitraps in the Gurunagar ward in Jaffna city during the COVID-19 lockdown period were decreased, with significantly lower proportions of Ae. aegypti than Ae. albopictus, compared with 2019.

\section{Conclusion}

Public health measures that restricted movement of people, closed schools, universities and offices in order to contain COVID-19 transmission unexpectedly led to a marked reduction in the incidence of dengue in Sri Lanka, in contrast to Singapore. The differences between the two tropical islands have significant implications for the epidemiology of dengue.

\section{Background}

The annual global burden of dengue has recently been estimated to be 390 million (95\% confidence interval or $\mathrm{Cl}$ of 284-528 million), with 96 million persons (95\% Cl of 67-136 million) manifesting clinical symptoms [1]. Approximately $70 \%$ of dengue cases occur in Asia. Aedes aegypti and Aedes albopictus are respectively the primary and secondary vectors of dengue and also vectors of other important arboviral diseases including chikungunya, yellow fever, Rift Valley fever and Zika worldwide [1-3].

Sri Lanka is a dengue-endemic tropical island in the Indian Ocean in proximity to South India and lying between latitudes $5^{\circ} 55^{\prime}$ and $9^{\circ} 51^{\prime} \mathrm{N}$ and longitudes $79^{\circ} 41^{\prime}$ and $81^{\circ} 53^{\prime} \mathrm{E}$. It has a population of 21.8 million, a land area of $65,525 \mathrm{~km}^{2}, 25$ administrative districts, and is separated by its central hills into dry and wet rainfall zones (Fig. 1). The wet zone, located in the hill country and the Southwest, receives an average annual rainfall of $250 \mathrm{~cm}$ in two main rainy seasons, the Northeast monsoon that normally occurs between October and December, and the Southwest monsoon that often begins in April and ends in June. Inter-monsoonal rains also occur between these periods in the wet zone. The dry zone, with an annual rainfall of $60-190 \mathrm{~cm}$, receives maximal rainfall during the Northeast monsoon and typically little or no 
rain for the rest of the year. An intermediate zone, with mixed characteristics, lies between the dry and wet zones (Fig. 1). The densely populated districts of Colombo, Gampaha and Kalutara are located in the wet zone, while the Jaffna district in the northern Jaffna peninsula lies in dry zone (Fig. 1). The Jaffna district, with Jaffna as its largest city (population 97,000 ), includes most of the peninsula and nearby islands, and has a land area of $1100 \mathrm{~km}^{2}$ with an average population density of approximately 700 persons $/ \mathrm{km}^{2}$. Although dengue has been present in Sri Lanka from the beginning of the 20th century, it has increased in prevalence throughout the island since the1990s with all four serotypes (DENV1-4) present and 105,049 cases in 2019 [4]. The established primary and secondary dengue vectors in Sri Lanka are also Aedes aegypti and Ae. albopictus respectively. Their abundance increases with monsoonal rains, and this is immediately followed by a surge in dengue cases [5-7].

Coronavirus disease 2019 (COVID-19) caused by the severe acute respiratory syndrome coronavirus 2 (SARS-CoV-2) was first detected in Wuhan, China in December 2019. COVID-19 has since spread rapidly to become a pandemic that caused approximately 168 million infections and 3.5 million deaths worldwide by 27 May 2021 [8], and severe social and economic disruption globally. The first case of COVID-19 in Sri Lanka was identified in January 2020. As of 27 May 2021, Sri Lanka is estimated to have had 172,277 cases and 1,298 deaths due to COVID-19 [8]. The first confirmed case of COVID-19 was recorded in the Jaffna district on 22 March 2020. Various public health measures (termed lockdown) to restrict COVID-19 transmission were introduced on 20 March 2020 in different districts, and these were relaxed or reimposed with changes in COVID-19 prevalence as shown in Table 1. A total of 42,702 and 122 COVID-19 cases in the year 2020, and 63,782 and 1,304 from January to the end of April 2021 were recorded island-wide and in the Jaffna district respectively [9]. 
Table 1

Timeline of the public health measures used for suppressing COVID-19 transmission in 2020 and 2021 in Sri Lanka

Period Measures Districts Affected

$1-1-2020$ to $18-\quad$ None
$3-2020$

18-3-2020 to Curfew, compulsory

Kalutara, Kandy and Puttalam

20-3-2020

wearing of face masks and maintenance of social

distancing

20-3-2020 to Nation-wide stay at home order All

20-4-2020

20-4-2020 to Curfew in selected districts Colombo, Gampaha, Kalutara, Kandy,

28-6-2020

20-4-2020 to Ban on inter-district travel All

Puttalam and Kegalle

26-5-2020

From 11-05- Partial opening of government establishments Colombo, Gampaha, and Kalutara

2020

26-5-20 to 28-6- Inter-district public movement allowed except in Colombo and Gampaha

$2020 \quad$ Colombo and Gampaha districts

From 06-06- Partial opening of schools All

2020

(grades 5, 11, and 13) excepting Colombo, Gampaha

and Kalutara districts

From 23-11- Partial opening of schools All

2020

(grade 6 to grade 13) except in Colombo, Gampaha and

Kalutara districts

29-10-2020 to Curfew in Colombo, Gampaha and Kalutara districts Colombo, Gampaha, and Kalutara

9-11-2020

1-11-2020 to 8- Restriction on inter-district travel for people in Colombo, Colombo, Gampaha and Kalutara

$11-2020$

Gampaha and Kalutara districts

12-12-2020 to Travel restrictions in selected areas of Galle district Galle

$18-12-2020$

From 11-01- All schools opened except in Colombo, Gampaha and Colombo, Gampaha and Kalutara $2021 \quad$ Kalutara districts

From 27-03- All schools and educational institutions closed All

$2021 \quad$ nationwide

Dengue is a disease that has to be notified by law to public health authorities of the Ministry of Health in Sri Lanka. Physicians in the state health sector follow the dengue management guidelines developed by the Ministry of Health [10]. In the absence of specific molecular diagnostic tests in many government hospitals, dengue is generally diagnosed based on clinical symptoms and other diagnostic tests. NS1 antigen detection along with serological assays are performed only where possible. Dengue fever and dengue hemorrhagic fever are considered in the differential diagnosis of patients presenting with acute onset of fever with the following signs/symptoms: headache, especially retro-orbital pain, myalgia /arthralgia, rash (diffuse, erythematous, macular) and hemorrhagic manifestations. A full blood count is performed to investigate leukopenia, thrombocytopenia and hematocrit. Generally, the presence of fever with at least two 
signs and symptoms mentioned above together with thrombocytopenia are considered sufficient for a diagnosis of dengue $[10,11]$.

The COVID-19 pandemic was expected to divert public health resources from dengue vector control programs, and thereby exacerbate dengue transmission [12]. In this context we analyzed the numbers of officially reported dengue cases in each of the 25 administrative districts of Sri Lanka from March 2020 to April 2021 inclusive, during which period public health measures were introduced to reduce COVID-19 transmission.

\section{Methods}

\section{Data on dengue and COVID-19 cases, and rainfall}

Monthly case data for dengue from the year 2015 until April 2021, and COVID-19 from March 2020 until the end of April 2021, were obtained from the Government of Sri Lanka Epidemiology Unit web site (https://www.epid.gov.lk/web/). Monthly rainfall data for the years 2017-2020 were obtained by request from the Government of Sri Lanka Meteorological Department and the 2021 data from its official website (https://www.meteo.gov.lk/index.php?lang=en).

\section{Predicted number of dengue cases during the COVID-19 lockdown period based on reported data for dengue cases from 2015 to 2019}

Even though public health measures to contain COVID-19 transmission were implemented since 18 March, 2020, because of epidemiological and rainfall data were only available on a monthly basis, the entire period 1 March 2020 to 30 April 2021 was used in our analysis as the lockdown period. Monthly dengue cases during the COVID-19 lockdown period were compared with the predicted number of monthly dengue incidence cases based on data from the prepandemic period from January 2015 to February 2020 using a Seasonal Autoregressive Integrated Moving Average (SARIMA) model described previously [13]. The SARIMA model is appropriate to do the seasonal time series analysis for the monthly dengue incidence data as the dengue incidence is seasonal and closely associated with monsoonal rainfall in Sri Lanka [5-7].

We established SARIMA models $(p, d, q) \times(P, D, Q)(p$ is the autoregressive lags, $d$ is the degree of differencing, $q$ is the moving-average lags, $P$ is the seasonal autoregressive lags, $D$ is the seasonal degree of differencing and $Q$ is the seasonal moving-average lags) for each district and for the whole island from the monthly reported dengue cases during the pre-pandemic period of 62 months from January 2015 to February 2020. SARIMA modelling and extraction of statistical parameters were performed using Python statistical libraries. A unique SARIMA $(1,1,0)(1,1,1)$ model with the strongest correlation of predicted and actual trends (mean $\mathrm{R}^{2}>0.7$ ), was selected from several models with different values of $(p, d, q) \times(P, D, Q)$ and the models from all the districts. The constructed SARIMA models used to predict dengue incidences for the 14 months from March 2020 to April 2021, during which period the different public health restriction measures shown in Table 1 were in place.

The predicted values and the actual values of dengue cases for Sri Lanka and each of the 25 districts were compared statistically for the 14 months (March 2020 to April 2021) of lockdown. Paired t-tests were conducted to determine the differences in means of reported monthly dengue cases and predicted dengue monthly cases for the whole of Sri Lanka and for each of the 25 districts. The null hypothesis of the t-test was that the means of the reported and predicted dengue cases were equal.

Rainfall and monthly numbers of dengue cases in the Jaffna district 
Monthly rainfall data from January 2015 to February 2020 and the reported monthly numbers of dengue cases from January 2015 to April 2021 and the SARIMA model-predicted numbers of monthly dengue cases from March 2020 to April 2021 in the Jaffna district were used to identify variations from the pattern observed in the pre-pandemic years of 2015 to 2019 that we had reported previously [7].

\section{Ovitrap collections and identification of Aedes larvae in Jaffna city}

Ovitrap-based Aedes larval collections were performed in Gurunagar ( $\left.9^{\circ} 39^{\prime} 12.6^{\prime \prime} \mathrm{N}, 80^{\circ} 01^{\prime} 03.5^{\prime \prime} \mathrm{E}\right)$, a coastal municipal ward in a densely populated residential area of Jaffna city from August 2020 to April 2021, essentially as done previously in Gurunagar in 2019 [7]. Conventional black plastic ovitraps (capacity: $650 \mathrm{ml}$, radius: $4.5 \mathrm{~cm}$, height: $10 \mathrm{~cm}$ ) containing $300 \mathrm{ml}$ of water obtained from the nearest domestic water supply (i.e. well or tap) with a $2 \times 15-\mathrm{cm}$ plywood paddle resting against the inside upper rim were placed outside different domestic residences as described [7]. Ten ovitraps were placed and fortnightly larval collections were made. Collected Aedes larvae were brought to the laboratory of the Department of Zoology, University of Jaffna. Larvae were maintained here under contained insectary conditions, and emerging adult mosquitoes identified at the species level with a standard key as previously described [7].

\section{Statistical analysis of Aedes larval collections from ovitraps}

Fisher's exact test was used to determine relationships between the number of positive ovitraps in 2019 and during the 2020/2021 lockdown period separately for Ae. aegypti and Ae. albopictus. Paired t-tests were performed to compare the numbers of Ae. aegypti and Ae. albopictus collected from ovitraps during 2019 and the 2020/2021 lockdown period.

\section{Results}

Dengue incidences during COVID-19 pre-pandemic and lockdown periods in Sri Lanka

The monthly predicted (during the 14 -month lockdown period between March 2020 and April 2021) and reported numbers of dengue cases (from January 2015 to April 2021) for the whole country is shown in Fig. 2. Peaks of dengue incidence follow soon after the two monsoons and the number of cases is also influenced by the location of the populous districts of Colombo, Gampaha and Kalutara in western Sri Lanka (identified by their principal cities in Fig. 1b) that are subject to both the Southwest and Northeast monsoons [6]. The total annual numbers of reported dengue cases for each of the 25 districts and the total for the whole country from January 2015 to April 2021 are shown in Additional file $\mathrm{S} 1$.

Statistical analysis employing the paired t-test revealed that the reported number of dengue cases for every month in the whole of Sri Lanka was significantly less than the predicted dengue incidences for the corresponding month (Additional file S2). The paired t-test also showed that the reported total number of dengue cases for the whole of Sri Lanka $(\bar{x}=843.76, \mathrm{sd}=1135.47)$ for the 14 -month lockdown period was significantly less $\left(\mathrm{t}_{(24)}=-5.036\right.$; $P$ one-tail t- test < $0.0001)$ than the predicted total number of dengue cases $(\bar{x}=3286.61, \mathrm{sd}=3044.94)$ for the country.

Dengue incidences during pre-pandemic and lockdown periods in each district of Sri Lanka

The total numbers of predicted and reported numbers of dengue cases during the 14-month lockdown period for each of the districts is illustrated graphically in Fig. 3.

Details of district-wise monthly and total reported and predicted dengue incidence during the 14-month lockdown period are provided in additional file S3. Detailed results of the statistical analysis using paired t-tests to compare means of monthly predicted and reported numbers of dengue cases for the 14-month lockdown period from March 2020 to April 2021 of 25 administrative districts of Sri Lanka are shown in Additional file S4. The statistical analysis revealed that the 
reported number of dengue cases was lower than the predicted number of dengue cases in all districts, and significantly so in all districts with the exception of the Batticaloa district (Additional file S4).

Dengue incidence in relation to rainfall and public health measures to control COVID-19 in the Jaffna district

Figure 4 shows the monthly numbers of reported and predicted dengue cases in association with monthly rainfall in the Jaffna district during the five pre-pandemic years and the lockdown period. Dengue incidence in the Jaffna district is seasonal and typically increases soon after the Northeast monsoon which prevails from October to December $[6,7]$. The rainfall pattern in 2020 was similar to that in the period 2015 to 2019 in the Jaffna district but the number of dengue cases reported in the Jaffna district declined in 2020 and first quarter of 2021 when compared with preceding years.

Aedes larvae collections in ovitraps from August 2020 to April 2021 in Jaffna city

In the period August 2020 to April 2021, a total of 90 and 282 Ae. aegypti and Ae. albopictus were respectively collected from the ten ovitraps in Gurunagar. A similar ovitrap collection during March - December 2019 in Gurunagar recorded 2380 and 1320 Ae. aegypti and Ae. albopictus respectively in nine ovitraps (details shown in Additional file S5). Both collections covered the Northeast monsoon of October- December, which is main rainy season in the Jaffna district due to its location in the dry zone of Sri Lanka. However, the collection in 2020 was begun in August 2020 which was approximately 15 weeks after the first introduction of COVID-19 containment measures in the country. Detailed collection data in 2020/2021 compared with 2019 (Additional file S5) show (i) a reduction in the monthly numbers of Aedes larvae collected in 2020 and 2021 compared with 2019; (ii) significantly decreased proportion of ovitraps with Ae. aegypti and Ae. albopictus larvae (Fisher's exact test $P<0.0001$ ) in 2020 and 2021 compared with 2019 and (iii) significantly reduced number of Ae. aegypti per ovitrap in comparison to Ae. albopictus $\left(\mathrm{t}_{(9)}=-2.42007, P\right.$ one-tailed t test $=0.0209$ ) during the lockdown period, whereas significantly greater numbers of Ae. aegypti than Ae. albopictus were collected from the ovitraps in 2019 at the same location [7]

\section{Discussion}

Numerous non-pharmaceutical public health measures were introduced early in the pandemic in almost all countries to suppress the spread of COVID-19 [14]. These have been supplemented with specific vaccination that became possible at the end of 2020. Sri Lanka was no exception and imposed lockdown immediately after an increase in number of cases in March 2020. The urgent need to control COVID-19 diverts resources away from other health sector activities, including the programs to control vector-borne diseases. One of the documented impacts has been on the significant increase in the incidence and mortality from malaria during the COVID-19 pandemic period in Zimbabwe [15]. On the other hand, it is expected that infectious diseases transmitted from person to person will decrease in incidence as a result of public health measures applied to reduce COVID-19 transmission. This is borne out by marked reductions in the incidence of influenza, a major respiratory disease, in diverse countries in both the northern and southern hemispheres [16-18].

The impact of COVID-19 containment measures on the incidence of dengue has been variable with reports of increases in Thailand and Singapore [19, 20] but a decrease in Malaysia [13]. Data from the second quarter of 2020 suggested that the incidence of dengue decreased in Sri Lanka during this period [21]. Our results for the whole of the lockdown period from March 2020 to December 2020 and first quarter of 2021 nationwide and district-wise, show a significant decrease in reported number of dengue cases compared with predictions based on the preceding five pre-pandemic years.

There is epidemiological evidence to suggest that movement of people outside of their homes is one of the factors that drive the transmission of dengue virus [22], and this is also supported by mathematical modeling studies [23]. In Sri Lanka dengue vectors are highly prevalent in premises of schools, hospital, government offices, transport hubs and 
factories [5, 7]. The anthropophagic Ae. aegypti and partly anthropophagic Ae. albopictus exhibit two peaks of blood feeding activity in the morning and afternoon, [24], that coincide with schooling and working hours. Our findings in the Jaffna district and the rest of Sri Lanka are therefore compatible with restrictions on movement of people leading to a reduction in dengue transmission $[22,23]$. However, other possible contributory factors need also to be considered: (i) Early COVID-19 and dengue manifest common clinical symptoms posing a problem for differential diagnosis [25-27]. It is therefore possible that the decrease in reported number of dengue cases in 2020 and 2021 during lockdown in Sri Lanka can also be due in part to the reluctance of people with mild dengue to seek medical treatment for fear of being identified as COVID-19 patients and quarantined; (ii) An important method for controlling dengue transmission in Sri Lanka is eliminating or minimising larval development habitats and application of larvicides by public health staff. However, these efforts were severely hampered by the COVID-19 containment measures in the Jaffna district and the rest of Sri Lanka and would therefore not have contributed to reducing the incidence of dengue in 2020 and 2021; (iii) Another possible factor is the decreased access to medical treatment (and therefore testing and recording of dengue) due to the different COVID-19 restrictions. It is likely that this also contributed to an unknown extent to the decrease in number of reported dengue cases; (iv) Movement restrictions of the population could have resulted in reduced access to human blood meals as Ae. aegypti and Ae. albopictus are both exophilic and Ae. aegypti is almost exclusively anthropophagic [28]. Diminished blood feeding will reduce oviposition and vector populations, particularly in the case of anthropophagic Ae. aegypti, which is compatible with the ovitrap collection results in Gurunagar, Jaffna city. In turn, this can reduce DENV transmission. More extensive vector data are however needed to firmly establish this possibility; ( $v$ ) Rainfall is the prominent factor influencing dengue transmission in Sri Lanka and the Jaffna district [6, 7]. The pattern of rainfall in the immediate pre-pandemic period in the whole of Sri Lanka as well as the Jaffna district was not strikingly different from that in 2020 and the first quarter of 2021. This does not support a role for rainfall variation in causing the reduced incidence of dengue in Sri Lanka in 2020 and early 2021.

Our findings from Sri Lanka are in contrast with data showing that dengue transmission increased during COVID-19 restrictions in Singapore [20]. Singapore, like Sri Lanka, is a tropical island. However, Singapore is a city state with many offices located in high rise buildings and robust measures in place to limit Aedes vector populations, so that public transport and work places are kept relatively free of Aedes. Sri Lanka, in contrast has a large rural area, a considerable rural working population, longer commuting distances for workers in large cities like Colombo and less effective vector control measures in many parts of the country, including the Jaffna district [7]. Public health interventions to contain COVID-19 in Singapore were also not identical to those applied in Sri Lanka. These factors may be responsible for the different impacts of COVID-19 restrictions on the incidence of dengue between Singapore and Sri Lanka.

\section{Conclusion}

During COVID-19 prevalence, a reduction in the numbers of reported dengue cases in comparison with that predicted from the preceding five-year pre-pandemic period was seen all 25 districts of Sri Lanka, with statistically significant reductions in 24 of the 25 districts. It is proposed that this may at least partly be due to public health measures that restricted movement of people in order to contain COVID-19 transmission resulting in reduced dengue transmission.

\section{Abbreviations}

COVID-19: coronavirus disease 2019; Cl: confidence interval; DENV - dengue virus; NS1: non structural protein 1; SARSCOV-2: severe acute respiratory syndrome corona virus 2

\section{Declarations}

\section{Availability of data and materials}


All data generated during this study are included in this published article and its additional files.

\section{Acknowledgements}

The field assistance of Public Health Officers is gratefully acknowledged.

\section{Funding}

Not applicable

\section{Authors' contributions}

SNS, RR: Conception and coordination; KS, SA, AT, TTPJ, TK, SNS: Investigation and data collection; SNS, RN, SR: Data analysis and image processing; SNS, RR: Writing the manuscript; All authors read and approved the final manuscript.

\section{Ethics approval and consent to participate}

Mosquitoes were collected in the field and reared in the insectary in accordance with the approved protocol of the Institutional Animal Ethics Committee of the University of Jaffna (AERC/2014/02). Ovitrap collections in private properties were carried out with verbal informed consent of owners of the property.

\section{Consent for publication}

Not applicable.

\section{Competing interests}

The authors declare that they have no competing interests.

\section{References}

1. World Health Organization. Dengue Fact Sheet. https://www.who.int/news-room/fact-sheets/detail/dengue-andsevere-dengue (2020). Accessed 25 May 2020.

2. Powell JR. Mosquito-borne human viral diseases: why Aedes aegypti? Am J Trop Med Hyg. 2018;98:1563-1565.

3. Leta S, Beyene TJ, De Clercq EM, Amenu K, Kraemer MUG, Revie CW. Global risk mapping for major diseases transmitted by Aedes aegypti and Aedes albopictus. Int J Infect Dis. 2018;67:25-35.

4. Epidemiology Unit, Ministry of Health, Sri Lanka. Dengue trends http://www.epid.gov.lk/web/index.php? option=com_content\&view=artic (2021). Accessed 13 May 2021.

5. Kusumawathie PHD. Larval infestation of Aedes aegypti and albopictus in six types of institutions in a dengue transmission area in Kandy, Sri Lanka. Dengue bull. 2005;29:165-168.

6. Surendran SN, Veluppillai T, Eswaramohan T, Sivabalakrishnan K, Noordeen F, Ramasamy R. Salinity tolerant Aedes aegyptiand albopictus-infection with dengue virus and contribution to dengue transmission in a coastal peninsula. J Vector Borne Dis. 2018;55:26-33.

7. Surendran SN, Jayadas TTP, Thiruchenthooran V, Raveendran S, Tharsan A, Santhirasegaram S, et al. Aedes larval bionomics and implications for dengue control in the paradigmatic Jaffna peninsula in northern Sri Lanka. Parasit Vectors. 2021;14:162.

8. Johns Hopkins University of Medicine, Coronavirus Resource Centre. https://coronavirus.jhu.edu/map.html (2021). Accessed 27 May 2021. 
9. Epidemiology Unit, Ministry of Health, Sri Lanka. COVID-19 updates https://www.epid.gov.lk/web/index.php? option=com_content\&view=article\&id=225\&lang=enn (2021). Accessed 13 May 2021.

10. Epidemiology Unit, Ministry of Health, Sri Lanka. Guidelines on management of dengue fever and dengue haemorrhagic fever in adults. National Guidelines. Revised and expanded edition 2012. Ministry of Health, Sri Lanka.

http://www.epid.gov.lk/web/images/pdf/Publication/guidelines_for_the_management_of_df_and_dhf_in_adults.pdf (2012). Accessed 29 Jan 2019.

11. Clinical Management of Dengue in Pregnancy. Ministry of Health Sri Lanka. https://slma.lk/wpcontent/uploads/2019/11/National-Guidelines-on-Clinical-Management-of-Dengue-Infection-in-Pregnancy-Reprintversion_new.pdf (2019). Accessed 25 Feb 2020.

12. Wilder-Smith A, Tissera H, Ooi EE, Coloma J, Scott TW, Gubler Preventing dengue epidemics during the COVID-19 pandemic. Am J Trop Med Hyg. 2020;103(2):570-571. doi: https://doi.org/10.4269/ajtmh.20-0480.

13. Ong SQ, Ahmad H, Mohd Ngesom AM. Implications of the COVID-19 lockdown on dengue transmission in Malaysia. Infect Dis Rep. 2021;13:148-159.

14. Wilder-Smith A, Freedman DO. Isolation, quarantine, social distancing and community containment: pivotal role for old-style public health measures in the novel coronavirus (2019-nCoV) outbreak. J Travel Med. 2020;27(2):taaa020. doi:1093/jtm/taaa020.

15. Gavi S, Tapera O, Mberikunashe J, Kanyangarara M. Malaria incidence and mortality in Zimbabwe during the COVID-19 pandemic: analysis of routine surveillance data. Malar J. 2021;20(1):233. doi:10.1186/s12936-02103770-7.

16. Cowling BJ, Ali ST, Ng TWY, Tsang TK, Li JCM, Fong MW, et al. Impact assessment of non-pharmaceutical interventions against coronavirus disease 2019 and influenza in Hong Kong: an observational study. Lancet Public Health. 2020;5(5):e279-e288. doi: 10.1016/S2468-2667(20)30090-6.

17. Sullivan SG, Carlson S, Cheng AC, Chilver MB, Dwyer DE, Irwin M, et al. Where has all the influenza gone? The impact of COVID-19 on the circulation of influenza and other respiratory viruses, Australia, March to September 2020. Euro Surveill. 2020;25(47):2001847. doi: 10.2807/1560-7917.ES.2020.25.47.2001847.

18. Zhang K, Misra A, Kim PJ, Moghadas SM, Langley JM, Smieja M. Rapid disappearance of influenza following the implementation of COVID-19 mitigation measures in Hamilton, Ontario. Can Commun Dis Rep. 2021;47(4):202-209. doi: $10.14745 /$ ccdr.v47i04a04.

19. Lim JT, Dickens BSL, Chew LZX, Choo ELW, Koo JR, Aik J, et al. Impact of SARSCoV-2 interventions on dengue transmission. PLoS Negl Trop Dis. 2020;14(10):e0008719.

20. Lim JT, Chew LZX, Choo ELW, Dickens BSL, Ong J, Aik J, et al. Increased dengue transmissions in Singapore Attributable to SARS-CoV-2 Social Distancing Measures. J Infect Dis. 2021;223(3):399-402.

21. Niriella MA, Ediriweera DS, De Silva AP, Premarathna BHR, Jayasinghe S, de Silva HJ. Dengue and leptospirosis infection during the coronavirus 2019 outbreak in Sri Lanka. Trans R Soc Trop Med Hyg. 2021:trab058.

22. Stoddard ST, Forshey BM, Morrison AC, Paz-Soldan VA, Vazquez-Prokopec GM, Astete H, et al. House-to-house human movement drives dengue virus transmission. Proc Natl Acad Sci USA. 2013;110(3):994-9. doi:10.1073/pnas.1213349110.

23. Falcón-Lezama JA, Martínez-Vega RA, Kuri-Morales PA, Ramos-Castañeda J, Adams B. Day-to-day population movement and the management of dengue epidemics. Bull Math Biol. 2016;78(10):2011-2033. doi: 10.1007/s11538-016-0209-6.

24. Paramasivan R, Philip Samuel P, Pandian RS. Biting rhythm of vector mosquitoes in a rural ecosystem of South India. Int J Mosq Res. 2015;2(3):106-113. 
25. Santoso MS, Masyeni S, Haryanto S, Yohan B, Hibberd ML, Sasmono RT, et al.Assessment of dengue and COVID-19 antibody rapid diagnostic tests cross-reactivity in Indonesia. Virol J. 2021;18:54.

26. Masyeni S, Santoso MS, Widyaningsih PD, Asmara DGW, Nainu F, Harapan H, et al. Serological cross-reaction and coinfection of dengue and COVID-19 in Asia: Experience from Indonesia. Int Jnfect Dis. 2021;102:152-154.

27. Guan WD, Ni DZ, Hu Y, Liang W, Ou C, He J, et al. Clinical characteristics of coronavirus disease 2019 in China. N Engl J Med. 2020;382:1708-1720. doi: 10.1056/NEJMoa2002032.

28. Myer MH, Fizer CM, Mcpherson KR, Neale AC, Pilant AN, Rodriguez A, et al. Mapping Aedes aegypti (Diptera: Culicidae) and Aedes albopictus vector mosquito distribution in Brownsville, TX. J Med Entomol. 2020;57:231-240. doi: 10.1093/jme/tjz132.

\section{Figures}

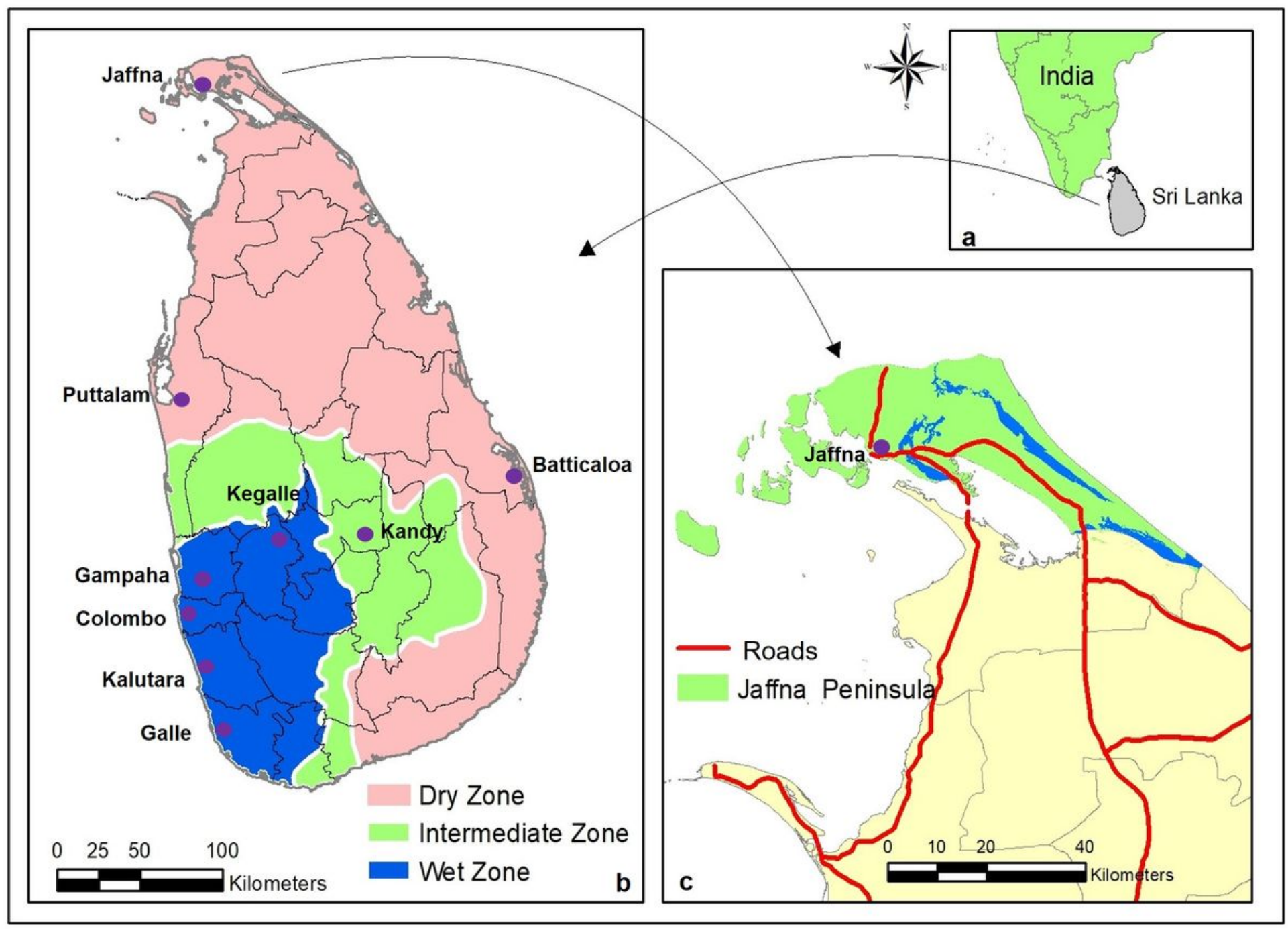

Figure 1

(a) Location of Sri Lanka in the Indian Ocean. (b) The 25 administrative districts and major cities in pertinent districts. The wet, intermediate and dry zones are demarcated. (c) Jaffna peninsula and Jaffna city in North Sri Lanka. 


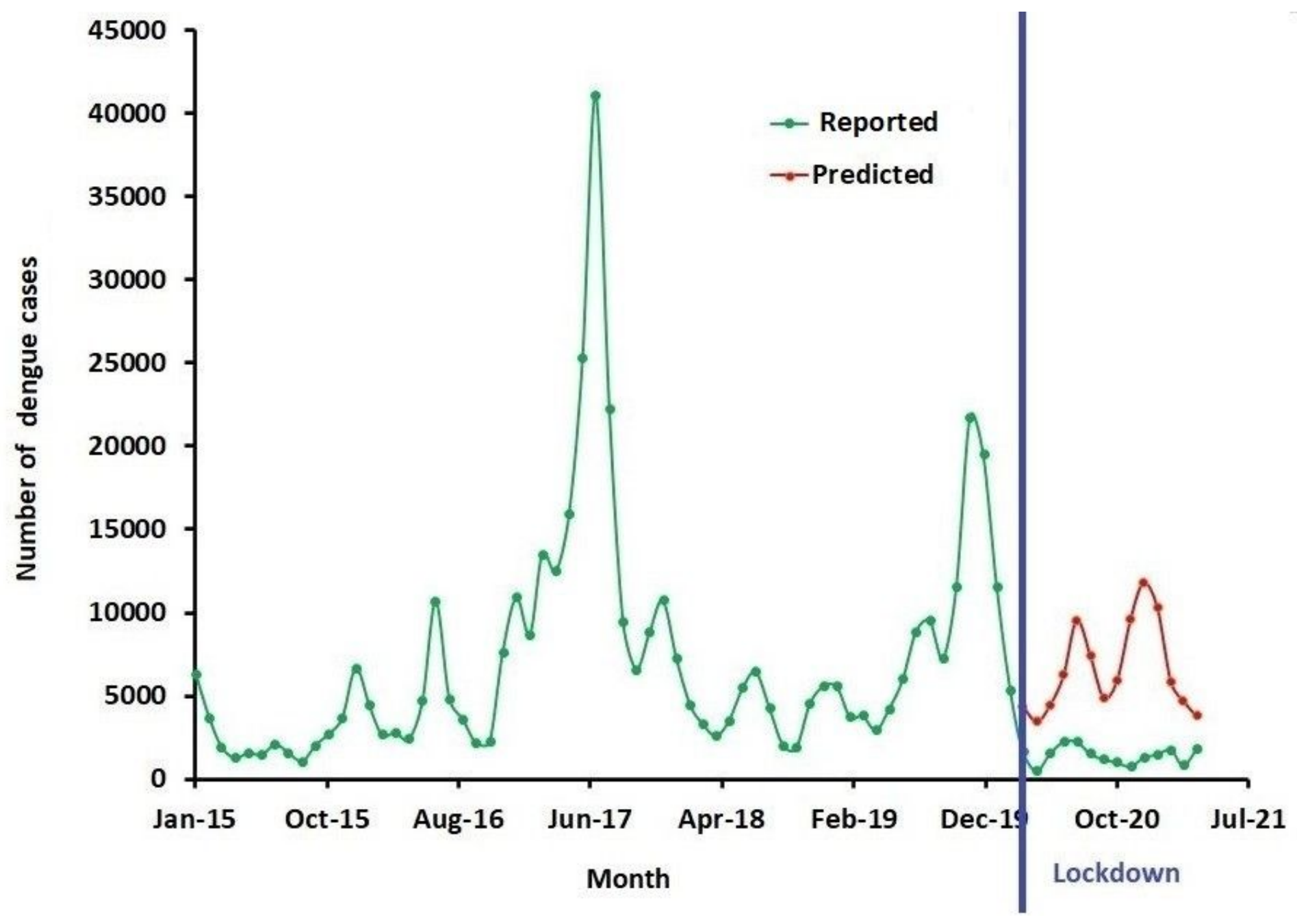

Figure 2

Reported and predicted numbers of monthly dengue cases for the whole of Sri Lanka. 


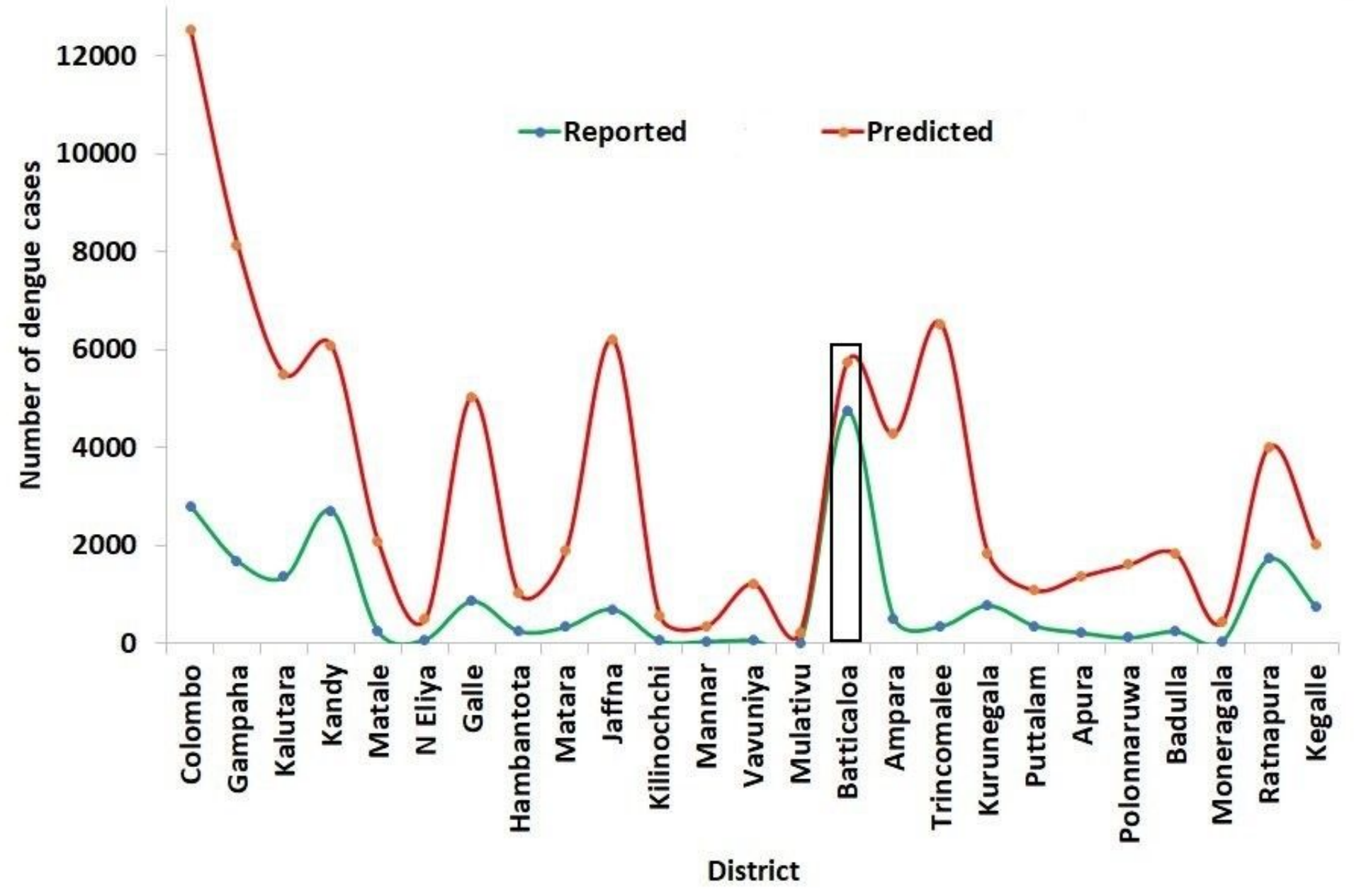

Figure 3

Reported and predicted numbers of dengue cases for each district from March 2020 to April 2021. 


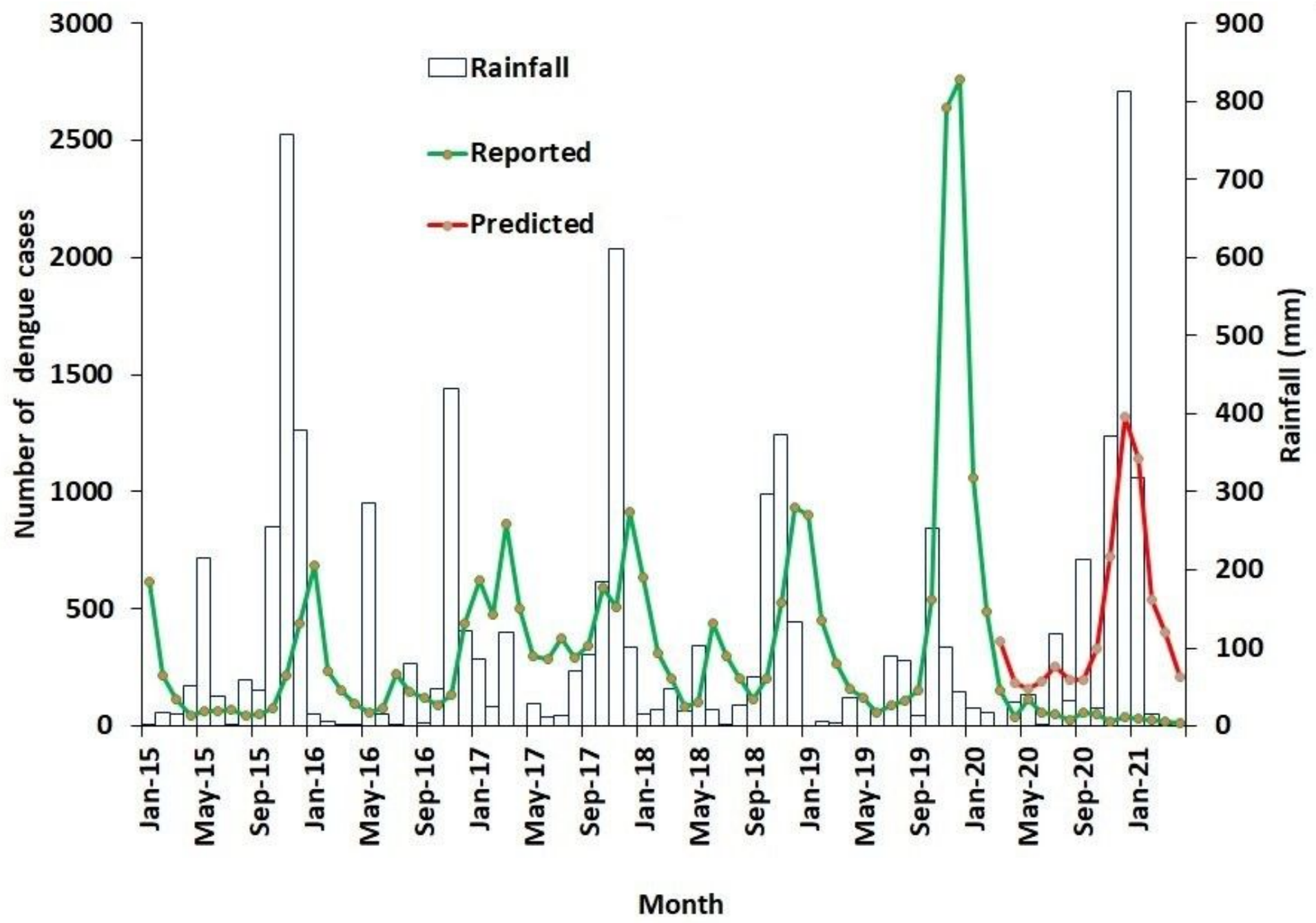

Figure 4

Reported and predicted numbers of monthly dengue cases and monthly rainfall in the Jaffna district.

\section{Supplementary Files}

This is a list of supplementary files associated with this preprint. Click to download.

- AdditionalfileS1.docx

- AdditionalfileS2.docx

- AdditionalfileS3.docx

- AdditionalfileS4.docx

- AdditionalfileS5.docx 